\title{
Novel Approach for Optimizing Information Propagation in Dynamic Social Network
}

\author{
SELVA KUMAR S ${ }^{1}$, KAYAVIZHY N ${ }^{2}$ \\ ${ }^{1}$ Assistant Professor, Department of Computer Science \& Engineering, BMSCE and affiliated to \\ Visvesvaraya Technological University, Belagavi, Karnataka, INDIA. \\ selva.cse@bmsce.ac.in \\ ${ }^{2}$ Associate Professor, Department of Computer Science \& Engineering \\ BMSCE and affiliated to Visvesvaraya Technological University, Belagavi, Karnataka, INDIA \\ kayarvizhyn.cse@bmsce.ac.in
}

\begin{abstract}
Identification of potential node is one of the essential operations to be carried out in social network analysis as it is necessary to undertake various important decisions associated with the information propagation. Review of existing literature towards social network highlights that there is very less work carried out towards emphasizing potential node. Therefore, the proposed study offers a novel and unique solution that is capable of optimizing the level of information propagation when it is exposed to dynamic networks. The proposed study has been modeled using graph theory and it uses degree centrality distribution in order to offer more insight towards analyzing the selection of potential nodes in a social network. The study significantly contributes towards precise information propagation and its sustainability in the presence of dynamic social network in every aspect.
\end{abstract}

Key-Words: - Information Propagation, Social Network Analysis, Potential Node, Influence maximization, centrality.

Received: September 5, 2019. Revised: December 10, 2019. Accepted: January 2, 2020. Published: January 23, 2020.

\section{Introduction}

Online Social Network has become a common platform to share and consume the data among the user. It also happens very dynamic in the network so that it's difficult to analyze the user behavior in such type of network. It attracted more attention from both industry and academia to solve many problems related to identifying the potential seed users [1]. With the advent of social networking, there is an increasing score of information that is shared and exchange among massive users with a higher degree of reliability [2]. Such forms of propagation of information and its trends are required for analyzing opinions and feedbacks that assists in formulating various critical decisions [3]. Therefore there is a strong involvement of potential nodes in social network analysis. The potential node is extremely essential for the purpose of decision making as it assists in studying the information flow over different forms of social media [4]. At present, there are various studies that are already carried out towards addressing different problems associated with the social network analysis [5]-
[8]. The analytical model proposed to gather the information dissemination dynamics of the social networks for analyzing advertisement and marketing purposes. The advertiser could use this model to adjust the speed of information propagation according to their strategies [9]. The distribution of the social network is studied with respect to multiple attributes centrality, bridge, density, strength, and structural holes. Out of all these attributes, centrality is one of the prominent attributes as it deals with quantifying the degree of influence associated with a specific node residing within a network and there are various ways this metric of centrality can be measured [10]. Usage of such principles is found to be adopted in mining, network modelling, sharing, and dynamic recommendation system, constructing analytics for social media, building business intelligence, etc. However, it is mainly targeted for developing applications for investigating the online behaviour of consumers. Its advance application uses collaborative learning.

Hence, this paper presents a novel approach for information propagation over a dynamic 
network using a unique optimization model. The proposed study enables computation of the closeness centrality to offer better information on the potential node as a matter of optimizing processing in a social network. The rest of the paper is organized as follows. The related work is summarized in Section 2. Section 3 discusses the adopted research methodology which is further followed by an elaborative discussion of algorithm design in Section 4. The framework of the proposed algorithm is presented in Section 5. Section 6 briefs of Results and discussion.

\section{Related Work}

Discussion on different forms of existing research towards information propagation has been carried out in our prior work [11]. Information propagation has been investigated with respect to the time factor in order to offer more dynamicity modeling in analysing propagation system [12]. Consideration of the time factor has also been seen in the work of (Goldenberg et al. [13]) where the authors presented scheduling seeding concept for improving the influence of a node in social networks. The usage of a clustering-based approach was also reported to enhance the identification of community which is required in precise information propagation [14]. Matteo et. al [15] Empirical study presents the factors enabling information propagation in Online Social Networks. Investigation towards anchor link offers a lot of significant information regarding information propagation in a social network [16]. Study towards securing the trust factor in social network significant affects information propagation and literature offers solutions to resists it [17]. Literature has also witnessed a classification method to discretize the level of friendships (Sever et al. [18]). Learning-based approaches are also used for analyzing the social network. The work of Rizi et al. [19] has used a deep learning approach in order to analyze comprehensive features associated with social network analysis. Chirag e. al. [20] presented a framework for examining the information propagation of certain topics in the blogosphere. This framework helps in identifying connectors and authorities in the given network. The usage of the clustering approach was carried out by Lan et al.[21] for promoting the beneficial feature of a multi-view clustering approach. Koufogiannis and Pappas [22] has discussed the propagation of malicious contents through the social network as a part of awareness study. The interest-matching users and multiple networks simultaneously were the two main factors validated using real-world data on Twitter-Foursquare and academic networks by Yilin et. al [23]. Udeep et. al. [24] proposed an approach to propagate the information in decentralized social networks to avoid propagating irrelevant data in a group. Naïve Bayes has utilized for forecasting different variants of links in order to address classification performance in social networks [25]. Krol and Atijas [26] have presented a study for identifying a specific community of friends. Machida et al. [27] have presented an approach for securing privacy problems in social networks. Arnaboldi et al.[28][29] have presented a discussion of using ego networks. Nick et al. [30] have presented a study to detect the possible relationship among the nodes in a network. Venkatanathan et al. [31] have investigated the empathy factor for social network analysis. Durr et al. [32] have investigated privacy problems in the social network considering a case study of the location. Doroud et al. [33] have used an arbitrary sampling of seed while Yun et al. [34] have modeled a secure system to safeguard the information propagation associated with a social network. The various studies show that information propagation to maximize the diffusion of influence in dynamic social networks. An algorithm and methods were developed to minimize the number of users or seeds with maximizing the influence and information diffusion in the social network within the given time bound [35-40]. Literature have also witnessed that researchers are looking towards the process of optimizing information propagation for various analyses. Optimizing the information propagation to maximize the information diffusion in uncertain networks, reach correct information to the right person on time and other applications as well [41][42]. 


\section{Proposed Description}

There has been various work carried out towards social network analysis, however, the study associated with the potential node exploration is quite less. The identification of potential nodes in the social network depends upon various extrinsic and intrinsic parameters. However, the research work in the present state doesn't emphasize on various factors associated with potential node exploration. Moreover, there is no standard framework or model that addresses such problems connected with potential nodes. Moreover, it is a highly computationally complex process to even model and process the massive set of distributed data in the social network for exploring potential node. Hence, the problem statement is "To develop a computational model that can optimize the level of information dissemination in dynamic network".

\section{Proposed Methodology}

The proposed work is a continuation of our prior work towards exploring potential node from social network analysis [43]. This part of the study is essentially meant for incorporating graph theory in order to optimize the search process towards potential nodes and exploring more interesting information associated with the potential nodes. The architecture of the proposed system is shown in Figure 1.

\begin{tabular}{|c|c|c|}
\hline \multicolumn{3}{|c|}{ Analytical Modelling } \\
\hline \multirow{7}{*}{$\begin{array}{l}\text { Network } \\
\text { Visualization } \\
\text { Process }\end{array}$} & & Connected Nodes \\
\hline & & Unconnected Nodes \\
\hline & \multicolumn{2}{|c|}{ Extraction of Potential Nodes } \\
\hline & \multicolumn{2}{|c|}{ Perform Ranking } \\
\hline & \multicolumn{2}{|c|}{ Distribution of Degree Centrality } \\
\hline & \multicolumn{2}{|c|}{ Computation by Shortest Paths } \\
\hline & \multicolumn{2}{|c|}{ Centrality of Closeness } \\
\hline
\end{tabular}

Figure 1 Proposed Architecture

The proposed system implements graph theory resulting in the exploration of connected/unconnected nodes. All the potential nodes are extracted from the connected nodes followed by sorting them and ranking them logic. The proposed system also implements the degree of centrality in order to investigate the possible influence of the potential nodes in social networks. Shortest paths are extracted from the graph in order to offer better information propagation followed by computation of centrality of the closeness; thereby showing interesting information of the potential node. The next section discusses the process of framework implementation.

\section{Framework Implementation}

The algorithm is designed on the basis of the principle that probability of improving the information dissemination could be significantly enhanced if the degree of dynamicity associated with the information could be addressed. At the same time, the data associated with social network differs from each other and therefore, the proposed a algorithm is designed in such a way that it could be applicable to any form of complex social network database.

\subsection{Strategy of Implementation}

The primary strategy of this algorithm implementation will target to implement a graph-based topology in order to map the complex structure of the social network. This strategy will assist in indexing the identified potential nodes and the edges connected through them. The secondary strategy of this algorithm will be to use the degree of centrality concept for the purpose of carrying out analysis of the edges that directly defines the stability of the flow of information propagation through them. The tertiary strategy of this algorithm will be to analyze the degree distribution and explore the best shortest links for faster information dissemination process. The adoption of these strategies in implementation will assist in optimizing the process of improving information propagation in social networks. 


\subsection{Algorithm for optimizing information dissemination}

This algorithm is responsible for carrying out the optimization operation towards the large scale social network database as input, which after processing will yield an outcome of the best potential nodes. The essential operational steps of the algorithm are as following:

\section{Algorithm for Optimizing Information Dissemination}

Input: $d b$ (social network database).

Output: top $_{\mathrm{m}}$ (sorted best $m$ potential nodes with relationship)

\section{Start}

$$
\begin{aligned}
& \text { 1. init } d b \\
& \text { 2. } \mathrm{C}_{\mathrm{E}} \rightarrow f_{1} \text { (edge); } \mathrm{C}_{\mathrm{EUG}} \rightarrow \mathrm{g}\left(\mathrm{C}_{\mathrm{E}}\right) \text {, } \\
& \mathrm{UC}_{\mathrm{N}} \rightarrow \operatorname{explore}\left(\operatorname{card}\left(\mathrm{C}_{\mathrm{EUG}}==0\right)\right) \\
& \text { 3. } \mathrm{C}_{\mathrm{EUG}} \rightarrow f\left(\mathrm{C}_{\mathrm{EUG}}, \mathrm{UC}_{\mathrm{N}}\right) \\
& \text { 4. } \operatorname{pot}_{\mathrm{n}} \rightarrow f_{3}\left(\operatorname{var}+1 \text {, unique }\left(\mathrm{C}_{\mathrm{E}}\right)\right. \\
& \text { 5. } \mathrm{PN}_{\mathrm{g}} \rightarrow f_{2} \text { (graph(idx), NC) } \\
& \text { 6. } \text { top }_{\mathrm{m}} \rightarrow \mathrm{PN}_{\mathrm{G}}(\operatorname{rank}(1: \mathrm{m})) \\
& \text { 7. } \mathrm{N} \rightarrow \operatorname{card}\left(\mathrm{C}_{\mathrm{EUG}}\right) \\
& \text { 8. [deg med_deg] } \rightarrow f(\operatorname{degPN}) \\
& \text { 9. } \mathrm{MD}_{\mathrm{G}} \rightarrow f\left(\operatorname{deg}_{\mathrm{G}}\right) \\
& \text { 10. [spath, d] } \rightarrow f_{4}\left(\mathrm{ON}_{\mathrm{G}}, \text { top }_{\mathrm{m}}\right) \\
& \text { 11. }\left[\mathrm{top}_{\mathrm{m}}\right] \rightarrow \operatorname{sort}(\mathrm{cc})
\end{aligned}
$$

\section{End}

Step-1 of the proposed algorithm takes the input of $d b$ (social network database) and then computes the communication edge $\left(\mathrm{C}_{\mathrm{E}}\right)$ as a unique value of sorted concatenated edge. In step- 2 the function $\mathrm{f}_{1}(\mathrm{x})$ is used for this purpose taking the input argument of edges obtained from the database $d b$. In a similar manner, undirected graph $\mathrm{C}_{\mathrm{EUG}}$ is constructed by applying function $g(\mathrm{x})$ over the obtained communication edge matrices $\mathrm{C}_{\mathrm{E}}$. Similarly, the proposed algorithm also constructs a matrix for all sorts of un-connected nodes $\mathrm{UC}_{\mathrm{N}}$.

$$
\mathrm{C}_{\mathrm{E}}=\left[\begin{array}{cc}
e 1,1 & e 2,1 \\
e 2,1 & e 2,2 \\
e m, 1 & e m, 2
\end{array}\right]
$$

The logic behind searching unconnected nodes is any nodes which deplete any form of communication edge of the undirected graph. Such forms of nodes are finally subjected to elimination shown in step-3. The algorithm constructs a function $f_{2}(\mathrm{x})$ and eliminates all the unconnected nodes $\mathrm{U}_{\mathrm{CN}}$ if they exist in $\mathrm{C}_{\mathrm{EUG}}$.

$$
\mathrm{UC}_{\mathrm{N}}=\operatorname{deg}\left(\mathrm{C}_{\mathrm{EUG}}==0\right)
$$

The next step of the algorithm implementation is to obtain the potential nodes by applying an explicit function $f_{3}(\mathrm{x})$. The function performs intersection operation between the matrix var with the identity of the potential node and matrix with the unique information of the communication edge $\mathrm{C}_{\mathrm{E}}$.

$$
\operatorname{pot}_{\mathrm{n}}=|\operatorname{var}| \bigcap|C e|
$$

The next part of the algorithm operation is associated with the selection of the potential node with respect to its identity. A new graph is constructed considering the identity obtained from the potential node in social network analysis followed by the degree of the potential nodes within the graph. The study considers the identification of the nodes that are poorly connected to each other followed by the elimination of such nodes. The algorithm looks for the condition of the degree of the potential node is found to be less than the certain threshold number (say 2), than the nodes are not considered to be connected to each other. Finally, a graph of potential nodes $\mathrm{PN}_{\mathrm{g}}$ is formed by the elimination of all the unconnected nodes from the graph of the potential nodes.

The next part of the algorithm is associated with exploring the elite outcome of the potential nodes from its graph and for this purpose; it makes use of the ranking mechanism. The ranking operation is carried out for $m$ number of the highest degree of the potential nodes $\mathrm{P}_{\mathrm{NG}}$ and the ranked information is stored in matrix top $\mathrm{p}_{\mathrm{m}}$. The consecutive part of the algorithm implementation is about extracting more information about the potential nodes. It does so by using a degree of centrality distribution (Eq.3) for analyzing the link quality. The potential node is always expected to offer a higher number of edges and hence exploration of the centrality distribution offers better insights to the potential node with respect to 
information propagation. The communication edges of the undirected graph $\mathrm{C}_{\mathrm{EUG}}$ are considered and the cardinality card is extracted and stored in matrix N. Therefore, the matrix N stores all the information of numbers of the nodes in the form of a graph. This operation is followed by the computation of the influence of potential nodes on a social network due to information propagation. This computation is carried out with an aid of degree deg and median of degree med_deg parameters. A function $f(\mathrm{x})$ is applied that computes median value associated with the degree of potential nodes.

$$
\mathrm{C}_{\mathrm{D}}(\mathrm{i})=\frac{C_{D}(i)}{N-1}
$$

The algorithm then computes similar median function $f(\mathrm{x})$ for the degrees of all the graphs. Finally, the algorithm performs the computation of the shortest path (Eq.4) $f_{4}(\mathrm{x})$ considering all the potential nodes involved in the graph with respect to top $\mathrm{p}_{\mathrm{m}}$ numbers. Finally, the $\operatorname{top}_{m}$ sorted information is retained in order to find a better relationship among the potential nodes in the form of distance among them. Hence, the proposed system offers better optimization towards potential node relationships.

$$
\sigma_{s t}(v)=\frac{N-1}{\sum_{t \in v} d_{G}(i, t)}
$$

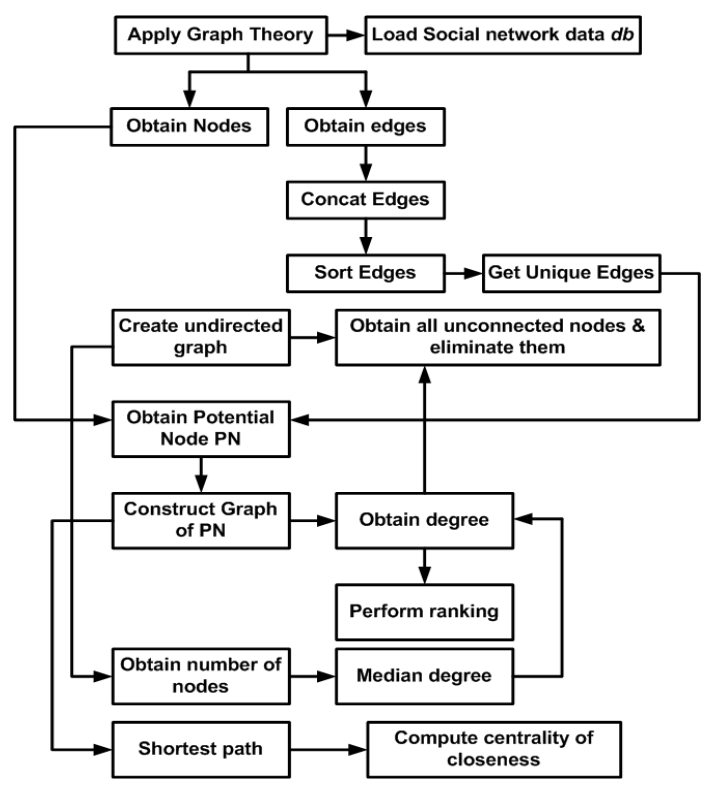

Figure 2 Flow of the Proposed Algorithm
A closer look into the proposed flow of the algorithm (Figure 2) highlights that it doesn't offer any form of a recursive operation which is one of the core features of this algorithm in order to perform optimization. Another significant aspect of this algorithm is that it offers optimization using simple graph logic as well as it doesn't use any form of external agents in order to obtain a better solution towards exploring interesting facts about potential nodes. Hence, the proposed system offers a better form of cost-effective architectural approach in order to ensure novel modeling of information propagation.

\section{Results Discussion}

The analysis of the proposed system is carried out using MATLAB considering the social network database from Stanford University [44]. The dataset consists of 4039 nodes and 88234 numbers of edges with a list of friends from social network Facebook. Different types of profiles that are on Facebook are considered as node feature along with ego networks and circle forms this dataset. The dataset has a mean coefficient of clustering as 0.6055 with 1612010 numbers of triangles.

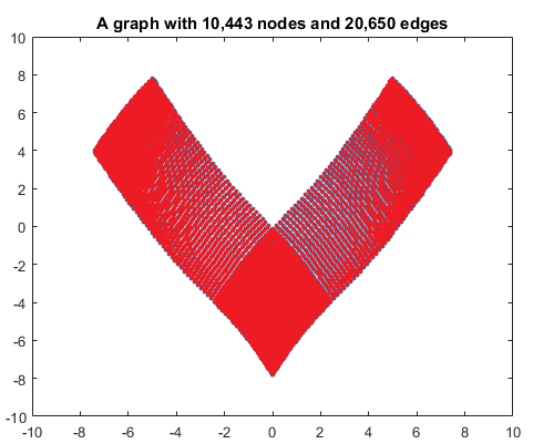

Figure 3 Visualization of Graph for the considered Facebook data [44]

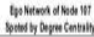

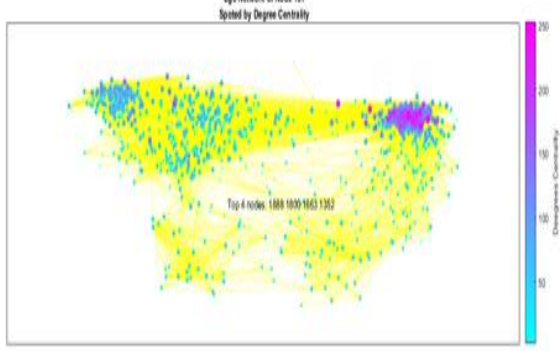

Figure 4 Visuals of Ego Network (Degree of Centrality) 
Figure 3 highlights the formation of the graph from 10,443 nodes and 20, 650 edges, which has been intentionally increased in order to perform analysis. The outcome shows clear visibility of the ego networks of a specific node that is spotted by the degree of centrality (Figure 4). The outcome highlights the comprehensive way of distinguishing the ego networks for the specific nodes for better identification of potential nodes with increased and decreased influence. Figure 5 highlights the degree distribution of the number of nodes with respect to the increasing degrees. The median degree found for this analysis is 36 for a specific node and 25 as a combo. Apart from this, the proposed system is also capable of significantly finding the shortest path for computing the closeness centrality. Figure 6 highlights a 4 hop distance between two specific nodes.

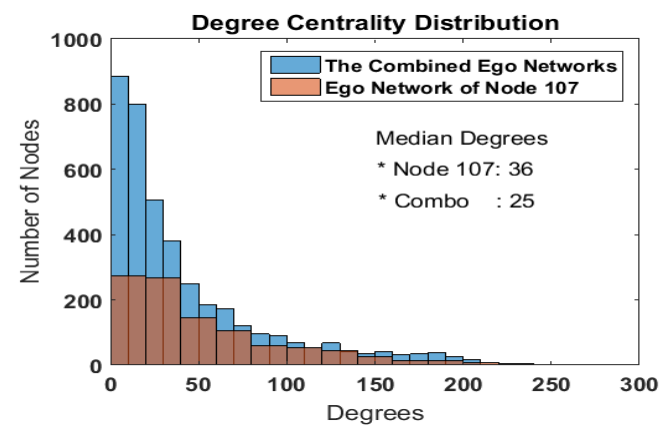

Figure 5 Degree Centrality Distribution

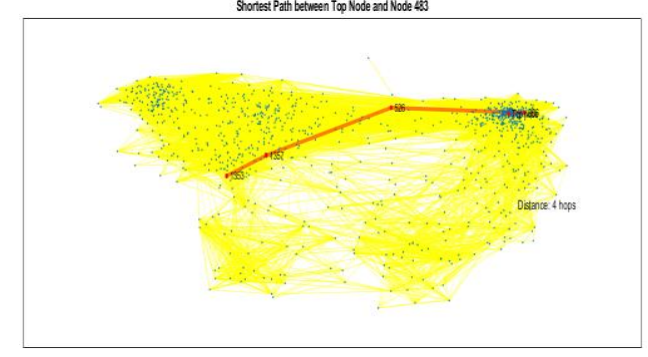

Figure 6 Visualization of Shortest Path

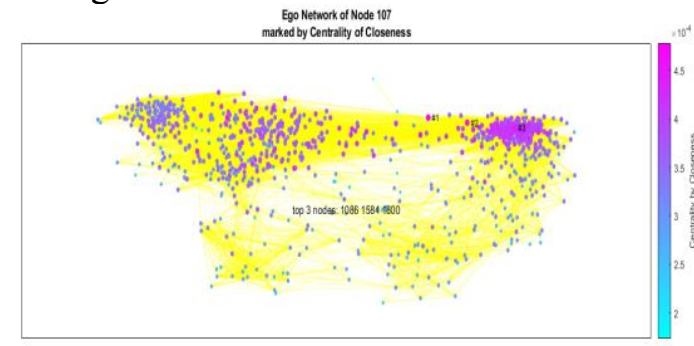

Figure 7 Ego Network (centrality of closeness)

Figure 7 highlights the ego network that offers impressively visualization of the specific node with the final outcome of top m-number of nodes (as per the algorithm). The complete processing just took 0.37761 seconds in core i3 processor, which is quite fast enough for performing processing operations.

\section{Conclusion}

This paper discusses the problems associated with the precise identification as well as the selection of the potential node in order to optimize the process of information dissemination. The contributions of the proposed study are as follows: i) the proposed study construct a dynamic network using a graph theory considering the massive number of nodes and edges for representing the large social network, ii) it also offers a flexible option to carry out degree distribution, iii) it introduces a mechanism to explore the shortest path between the specific target node and top node, iv) the proposed study also enables computation of the closeness centrality to offer better information on the potential node as a matter of optimizing processing in the social networks.

\section{Acknowledgement}

We gratefully thank the Visvesvaraya Technological University, Jnana Sangama, Belagavi for financial support extended to this research work.

\section{References}

[1] Fast Information Propagation In Social Networks Feng Zou-James Willson-Zhao Zhang-Weili $\mathrm{Wu}$, Discrete Mathematics, Algorithms and Applications, 2010

[2] Nilanjan Dey, Rosalina Babo, Amira S. Ashour, Vishal Bhatnagar, Med Salim Bouhlel, "Social Networks Science: Design, Implementation, Security, and Challenges: From Social Networks Analysis to Social Networks Intelligence", Springer-Computer, 2018

[3] Mehmet Kaya, Jalal Kawash, Suheil Khoury, Min-Yuh Day, "Social Network Based Big Data Analysis and Applications", Springer, 2018

[4] Xinyue Ye, Xingjian Liu, Cities as Spatial and Social Networks, Springer, 2018

[5] Y. Li, J. Fan, Y. Wang and K. Tan, "Influence Maximization on Social Graphs: A Survey," in 
IEEE Transactions on Knowledge and Data Engineering, vol. 30, no. 10, pp. 1852-1872, 1 Oct. 2018.

[6] H. Falk, "Applications, architectures, and protocol design issues for mobile social networks: A survey," in Proceedings of the IEEE, vol. 99, no. 12, pp. 2125-2129, Dec. 2011.

[7] M. A. Hannah and M. Simeone, "Exploring an Ethnography-Based Knowledge Network Model for Professional Communication Analysis of Knowledge Integration," in IEEE Transactions on Professional Communication, vol. 61, no. 4, pp. 372-388, Dec. 2018.

[8] B. Lauren and S. Pigg, "Networking in a Field of Introverts: The Egonets, Networking Practices, and Networking Technologies of Technical Communication Entrepreneurs," in IEEE Transactions on Professional Communication, vol. 59, no. 4, pp. 342-362, Dec. 2016.

[9] Y. Chou, H. Huang and R. Cheng, "Modeling Information Dissemination in Generalized Social Networks," in IEEE Communications Letters, vol. 17, no. 7, pp. 1356-1359, July 2013.doi:10.1109/LCOMM.2013.052013.1300

[10] Natarajan Meghanathan, Centrality Metrics for Complex Network Analysis: Emerging Research and Opportunities, IGI Global, 2018

[11] Selva Kumar S and Dr. Kayarvizhy N, "A Comprehensive Insight towards Research Direction in Information Propagation" International Journal of Advanced Computer Science and Applications(IJACSA), 8(4), 2017. http://dx.doi.org/10.14569/IJACSA.2017.08040 6

[12] Y. Wu, H. Huang, J. Zhao, C. Wang and T. Wang, "Using Mobile Nodes to Control Rumors in Big Data Based on a New Rumor Propagation Model in Vehicular Social Networks," in IEEE Access, vol. 6, pp. 6261262621, 2018.

[13] D. Goldenberg, A. Sela and E. Shmueli, "Timing Matters: Influence Maximization in Social Networks Through Scheduled Seeding," in IEEE Transactions on Computational Social Systems, vol. 5, no. 3, pp. 621-638, Sept. 2018.

[14] M. H. Hussein, H. N. Nawaf and W. S. Bhaya, "Exploiting the shared neighborhood to improve the quality of social community detection," 2017 Annual Conference on New Trends in Information \& Communications Technology Applications (NTICT), Baghdad, 2017, pp. 52-56.
[15] M. Magnani, D. Montesi and L. Rossi, "Information Propagation Analysis in a Social Network Site," 2010 International Conference on Advances in Social Networks Analysis and Mining, Odense, 2010, pp. 296-300. doi: 10.1109/ASONAM.2010.62

[16] J. Ma et al., "Balancing User Profile and Social Network Structure for Anchor Link Inferring Across Multiple Online Social Networks," in IEEE Access, vol. 5, pp. 1203112040, 2017.

[17] M. Samanta, P. Pal and A. Mukherjee, "Prevention of information leakage by modulating the trust uncertainty in EgoNetwork," 2017 9th International Conference on Communication Systems and Networks (COMSNETS), Bangalore, 2017, pp. 377-378.

[18] N. Sever, L. Humski, J. Ilić, Z. Skočir, D. Pintar and M. Vranić, "Applying the multiclass classification methods for the classification of online social network friends," 2017 25th International Conference on Software, Telecommunications and Computer Networks (SoftCOM), Split, 2017, pp. 1-6.

[19] F. S. Rizi, M. Granitzer and K. Ziegler, "Global and Local Feature Learning for EgoNetwork Analysis," 2017 28th International Workshop on Database and Expert Systems Applications (DEXA), Lyon, 2017, pp. 98-102.

[20] Chirag Shah, "Information Derivatives - A New Way to Examine Information Propagation”, HCIR 2010, August 22, 2010, New Brunswick, NJ, USA.

[21] C. Lan, Y. Yang, X. Li, B. Luo and J. Huan, "Learning Social Circles in Ego-Networks Based on Multi-View Network Structure," in IEEE Transactions on Knowledge and Data Engineering, vol. 29, no. 8, pp. 1681-1694, 1 Aug. 2017.

[22] F. Koufogiannis and G. J. Pappas, "Diffusing Private Data Over Networks," in IEEE Transactions on Control of Network Systems, vol. 5, no. 3, pp. 1027-1037, Sept. 2018.

[23] Yilin Shen, Thang N. Dinh, Huiyuan Zhang, and My T. Thai. 2012. Interest-matching information propagation in multiple online social networks. In Proceedings of the $21 \mathrm{st}$ ACM international conference on Information and knowledge management (CIKM '12). ACM, New York, NY, USA, 1824-1828. DOI: https://doi.org/10.1145/2396761.2398525

[24] Tandukar U., Vassileva J. (2012) Selective Propagation of Social Data in Decentralized 
Online Social Network. In: Ardissono L., Kuflik T. (eds) Advances in User Modeling. UMAP 2011. Lecture Notes in Computer Science, vol 7138. Springer, Berlin, Heidelberg

[25] A. K. Gupta and N. Sardana, "Naïve Bayes Approach for Predicting Missing Links in Ego Networks," 2016 IEEE International Symposium on Nanoelectronic and Information Systems (iNIS), Gwalior, 2016, pp. 161-165.

[26] D. Król and S. Atijas, "Common Features against Similarity for Discovering Social Circles in Networks," 2015 Second European Network Intelligence Conference, Karlskrona, 2015, pp. 91-97.

[27] S. Machida, T. Kajiyama, S. Shigeru and I. Echizen, "Analysis of Facebook Friends Using Disclosure Level," 2014 Tenth International Conference on Intelligent Information Hiding and Multimedia Signal Processing, Kitakyushu, 2014, pp. 471-474.

[28] V. Arnaboldi, M. Conti, A. Passarella and F. Pezzoni, "Ego networks in Twitter: An experimental analysis," 2013 Proceedings IEEE INFOCOM, Turin, 2013, pp. 3459-3464.

[29] Arnaboldi, Valerio, Marco Conti, Andrea Passarella, and Fabio Pezzoni. "Analysis of ego network structure in online social networks." In Privacy, security, risk and trust (PASSAT), 2012 international conference on and 2012 international confernece on social computing (SocialCom), pp. 31-40. IEEE, 2012.

[30] B. Nick, C. Lee, P. Cunningham and U. Brandes, "Simmelian backbones: Amplifying hidden homophily in Facebook networks," 2013 IEEE/ACM International Conference on Advances in Social Networks Analysis and Mining (ASONAM 2013), Niagara Falls, ON, 2013, pp. 525-532.

[31] J. Venkatanathan, E. Karapanos, V. Kostakos and J. Gonçalves, "A network science approach to Modelling and predicting empathy," 2013 IEEE/ACM International Conference on Advances in Social Networks Analysis and Mining (ASONAM 2013), Niagara Falls, ON, 2013, pp. 1395-1400.

[32] M. Dürr, M. Maier and K. Wiesner, "An Analysis of Query Forwarding Strategies for Secure and Privacy-Preserving Social Networks," 2012 IEEE/ACM International Conference on Advances in Social Networks Analysis and Mining, Istanbul, 2012, pp. 535542.

[33] M. Doroud, P. Bhattacharyya, S. F. Wu and D. Felmlee, "The Evolution of Ego-Centric Triads: A Microscopic Approach toward
Predicting Macroscopic Network Properties," 2011 IEEE Third International Conference on Privacy, Security, Risk and Trust and 2011 IEEE Third International Conference on Social Computing, Boston, MA, 2011, pp. 172-179

[34] X. Yun, S. Li and Y. Zhang, "SMS Worm Propagation Over Contact Social Networks: Modeling and Validation," in IEEE Transactions on Information Forensics and Security, vol. 10, no. 11, pp. 2365-2380, Nov. 2015.

[35] G. Tong, W. Wu, S. Tang and D. Du, "Adaptive Influence Maximization in Dynamic Social Networks," in IEEE/ACM Transactions on Networking, vol. 25, no. 1, pp. 112-125, Feb. 2017. doi: 10.1109/TNET.2016.2563397

[36] Q. Liqing, Y. Jinfeng, F. Xin, J. Wei and G. Wenwen, "Analysis of Influence Maximization in Temporal Social Networks," in IEEE Access, vol. 7, pp. 42052-42062, 2019. doi: 10.1109/ACCESS.2019.2894155

[37] D. Jing and T. Liu, "Structural Influence Maximization in Social Networks," 2019 International Conference on Internet of Things (iThings) and IEEE Green Computing and Communications (GreenCom) and IEEE Cyber, Physical and Social Computing (CPSCom) and IEEE Smart Data (SmartData), Atlanta, GA, USA, 2019, pp. 1088-1095. doi:

10.1109/iThings/GreenCom/CPSCom/SmartDat a.2019.00186

[38] Y. Mei, W. Zhao and J. Yang, "Maximizing the Effectiveness of Advertising Campaigns on Twitter," 2017 IEEE International Congress on Big Data (BigData Congress), Honolulu, HI, 2017, pp. 73-80. doi: 10.1109/BigDataCongress.2017.19

[39] G. Wang, J. Jiang, W. Li and C. Wang, "Influence Maximization Based on Node Attraction Model," 2019 IEEE Intl Conf on Dependable, Autonomic and Secure Computing, Intl Conf on Pervasive Intelligence and Computing, Intl Conf on Cloud and Big Data Computing, Intl Conf on Cyber Science and Technology Congress (DASC/PiCom/CBDCom/CyberSciTech),

Fukuoka, Japan, 2019, pp. 437-441. doi:

10.1109/DASC/PiCom/CBDCom/CyberSciTec h.2019.00089

[40] V. K. Yalavarthi and A. Khan, "Steering Top-k Influencers in Dynamic Graphs via Local Updates," 2018 IEEE International Conference 
on Big Data (Big Data), Seattle, WA, USA, 2018, pp. 576-583. doi: 10.1109/BigData.2018.8621873

[41] C. Frey, A. Züfle, T. Emrich and M. Renz, "Efficient Information Flow Maximization in Probabilistic Graphs (Extended Abstract)," 2018 IEEE 34th International Conference on Data Engineering (ICDE), Paris, 2018, pp. 18011802.

doi: 10.1109/ICDE.2018.00258

[42] Chen DB, Wang GN, Zeng A, Fu Y, Zhang YC. Optimizing Online Social Networks for Information Propagation. PloS one 2014; 9: e96614. pmid:24816894

[43] Shekar, Selva Kumar \& Nagappan, Kayarvizhy \& Rajendran, Balaji. (2017). SADI: Stochastic Approach to Compute Degree of Importance in Web-Based Information Propagation. 10.1007/978-3-319-57141-6_36.

[44] "Snap-Standard", https://snap.stanford.edu/data/, Retrieved on 1710-2019 